\title{
Québec et ses dilemmes urbanistiques : les enseignements du terrain et des débats médiatiques
}

\author{
Martin Simard ${ }^{\mathrm{a}}$
}

RÉSUMÉ. Les villes d'une certaine taille sont constamment interpellées par des enjeux ou projets urbanistiques de différentes natures et dimensions. Québec vit actuellement une période de croissance, et plusieurs dilemmes urbains se présentent simultanément dans la sphère publique et se télescopent, dans une certaine mesure. Ces dilemmes se démarquent par la taille des projets et des investissements requis ainsi que par l'ampleur des effets pressentis. Les délibérations sur ces dilemmes sont intéressantes dans le sens où elles font ressortir différentes questions théoriques dans le champ de l'urbanisme. Nous traiterons donc de ces interrogations à l'aide d'une revue de presse sur les trois dilemmes identifiés, de quelques traitements statistiques et cartographiques ainsi que d'un retour sur les principes fondamentaux du champ de l'urbanisme qui sont mis à l'épreuve sur le terrain.

\begin{abstract}
Large cities are constantly challenged by planning issues that are different in nature and prospect. Quebec City is currently experiencing a period of growth, and several urban dilemmas emerge simultaneously in the public sphere and are telescoped, to a certain extent. These dilemmas are distinguished in project scope, investment sums required and magnitude of the anticipated effects. The debates on these dilemmas are interesting in that they bring out different theoretical questions in urban planning. We will therefore deal with these issues and analyse them via a press review, some statistical and cartographic processing, and by reverting to the fundamental principles in urban planning that are tested in the field.
\end{abstract}

\section{Introduction}

Les villes d'une certaine taille sont constamment interpellées par des enjeux ou projets urbanistiques de différentes natures et dimensions. Dans une économie de marché, le développement urbain au sens large (immobilier, industriel et commercial) met en mouvement une foule d'intervenants privés qui mettent constamment en branle des stratégies spatiales susceptibles de modifier l'environnement bâti (Guy et Henneberry, 2000). De leur côté, les organismes gouvernementaux conçoivent des projets d'infrastructures ou d'équipements publics en fonction des demandes et des besoins réels ou perçus. Ces enjeux ou projets doivent être conçus, arbitrés et/ou autorisés par les administrations locales en vertu de leur responsabilité en matière d'urbanisme (Hodge et Gordon, 2013). En fait, il serait peut-être plus juste de parler de dilemmes, car les interventions en aménagement et en urbanisme comportent toujours une série de conséquences positives ou négatives qu'il convient de soupeser, quelle que soit la décision finale (Dorning, Koch, Shoemaker et Meentemeyer, 2015).

Québec vit actuellement une période de croissance, et plusieurs dilemmes urbains se présentent simultanément dans la sphère publique et se télescopent, dans une certaine mesure. Ces dilemmes se démarquent par la taille des projets et des investissements requis ainsi que par l'ampleur des effets pressentis et par le fait qu'ils soient promus ou appuyés par divers acteurs, notamment les gouvernements provincial et fédéral, voire la municipalité elle-même. Après les efforts consentis entre 1990 et 2010 à la revitalisation du quartier

a Professeur, membre du Centre de recherche sur le développement territorial (CRDT), Université du Québec à Chicoutimi 
Saint-Roch (Leclerc, 2018), à la densification résidentielle et commerciale (Mercier et Roy, 2014) et aux projets variés associés aux fêtes du 400 e anniversaire $^{1}$ et à l'aménagement de la promenade Samuel-De-Champlain. Les enjeux actuels concernent 1) le projet de réseau de tramway, 2) la construction d'un éventuel 3 e lien entre les rives du fleuve Saint-Laurent et 3) l'érection d'une tour à bureaux titanesque dénommée Le Phare²

Les délibérations sur ces dilemmes qui se produisent dans la sphère médiatique, ou qui sont rapportées dans celle-ci, sont intéressantes dans le sens où elles font ressortir différentes questions théoriques dans le champ de l'urbanisme. À titre d'exemples, mentionnons le rôle des statistiques et des rapports d'experts, les effets des consultations publiques de même que les rapports de pouvoir entre les diverses instances gouvernementales dans les processus de planification et de prise de décision. À cet égard, le terrain et les débats urbanistiques sur la scène locale nous permettent des réflexions fondamentales, au-delà de leurs aspects factuels. Incidemment, cette micropolitique du lieu qui est sous-jacente à l'urbanisme mériterait davantage d'attention, selon certains analystes (Beauregard, 2013).

Nous traiterons donc de ces interrogations à l'aide d'une revue de presse sur les trois dilemmes identifiés ainsi que de quelques traitements statistiques et cartographiques. En ce qui concerne le cheminement des idées, nous mettrons d'abord en contexte l'agglomération ${ }^{3}$ de Québec, avant de présenter les dilemmes ciblés et de discuter des dimensions théoriques qui peuvent y être décelées. Ces dimensions seront analysées en quatre temps, c'est-à-dire le caractère politique de l'urbanisme, l'idéal de la participation et des consultations publiques, l'importance des études pour supporter les interventions de même que les capacités prévisionnelles de l'urbanisme de planification.

\section{Québec : une mise en contexte}

Première ville d'origine européenne au nord du fleuve Rio Grande, capitale du Québec et, temporairement, du Canada-Uni, ville du patrimoine mondial, Gilbraltar d'Amérique (Noppen, Morisset et Karam, 2008), voire enclave surréaliste (Boyer, 2019), Québec porte plusieurs surnoms ou définitions. Toutefois, on peut affirmer plus simplement qu'elle constitue un établissement urbain francophone situé sur la rive nord du fleuve Saint-Laurent et qu'elle a donné son nom à la province canadienne fondée sur les vestiges de la Nouvelle-France.

Tout d'abord, Québec est une agglomération marquée par son site et par sa situation (Blanchard, cité par Grenier, 2008). En effet, le relief et l'hydrographie aux propriétés défensives ont favorisé sa fondation, mais également l'émergence de la haute-ville et de la basse-ville, affectant du coup sa structure urbaine autant que sa géographie sociale. De plus, Québec a vécu des périodes de croissance et de stagnation sur les plans démographique et économique (Vallières, 2010). Pôle administratif, intellectuel et religieux aux XVII e et

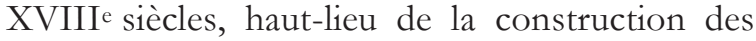
bateaux de bois au XIX ${ }^{\text {e }}$ siècle, la ville sera dans l'ombre de Montréal au moment de l'essor de l'industrialisation (1870-1950).

Il faudra attendre l'expansion de l'État québécois, dans les années 1960, pour que Québec vive une croissance urbaine significative. L'expansion de la colline Parlementaire et la construction d'un réseau d'autoroutes caractérisent cette époque (Lebel, 2005). Le processus de modernisation véhicule son lot d'interventions ultérieurement jugées comme des erreurs urbanistiques (Mercier, 2000), par exemple la construction de l'édifice Marie-Guyard (ou Complexe G) près de l'Assemblée nationale (voir figure 1), les démolitions liées à la «rénovation urbaine» dans les quartiers anciens ou la «bétonisation de la rivière SaintCharles » (Boutin, 2008). Avec la Révolution tranquille et la montée du nationalisme québécois, Québec devient aussi le symbole de la culture française en Amérique. Paradoxalement, la ville comporte un grand nombre de propriétés fédérales, notamment le Port de Québec, la base militaire de Valcartier et le parc commémoratif des Champs-de-Bataille. 




Figure 1- L'édifice Marie-Guyard, aussi appelé le Complexe G

Source : Martin Simard, 2015

Son homogénéité ethnique, son conservatisme et son ancrage dans le Québec profond lui ont valu le surnom de "Vieille Capitale», une image qui contraste avec celle de Montréal, métropole cosmopolite manifestement intégrée à la modernité nord-américaine (Racine et Villeneuve, 1992). Cette image patrimoniale de Québec serait en partie surfaite, considérant la modernisation des années d'après-guerre selon le trio voiture-bungalowcentre commercial (Mercier, 2010). D'autres vont jusqu'à dire que le Vieux-Québec est une sorte de Disneyland entouré d'une marée de pavillons de banlieue (Geronimi, 2008). Faut-il se surprendre qu'une copie du Vieux-Québec sera conçue en Chine, à proximité de Shanghai, en 2022 (Siou, 2017)? L'original et la future copie soulèvent paradoxalement tous deux des interrogations en matière d'authenticité.
Quoi qu'il en soit, depuis les fêtes du 400e anniversaire, survenues en 2008, Québec démontre un dynamisme qui tend à contredire sa "personnalité géographique » traditionnelle de grande ville de province. En effet, la population croît et se diversifie à bon rythme, le chômage est au plus bas et le développement urbain y est vigoureux. Qualifiée de « mystère de Québec » (Polèse, 2012), cette période de croissance s'appuie notamment sur le développement de la haute technologie (multimédia, optique, etc.). En voie de devenir une métropole, la région métropolitaine de recensement (RMR) de Québec s'étale maintenant sur les deux rives du fleuve Saint-Laurent et déborde la plaine du même nom pour prendre d'assaut les contreforts du massif laurentien, sur la rive nord (voir figure 2). 




Figure 2 - Le territoire de la RMR de Québec Source : Statistique Canada, 2016a

La RMR de Québec avait une population de 800296 en 2016, selon les données du recensement canadien, soit une augmentation de 4,3\% entre 2011 et $2016^{4}$. Il s'agit de la $2^{\mathrm{e}}$ plus grande zone urbaine au Québec et de la 7e au Canada (Statistique Canada, 2016b). Malgré les tendances nouvelles à la diversification, la population de la RMR de Québec demeure très homogène, avec un taux de $94,8 \%$ de gens de langue maternelle française et de $93,6 \%$ de non-immigrants, selon la terminologie des statisticiens (Stastistique Canada, 2016b). Ces pourcentages illustrant l'homogénéité sociale sont exceptionnels. Aucune autre agglomération de plus de 500000 habitants au Canada, et probablement aux États-Unis, ne comporte si peu d'immigrants ou de personnes de langue maternelle autre que celle de la majorité (voir tableau 1$)^{5}$. Il y a des débats récurrents sur la cause de cette situation (Bourque, 2019). Pour notre part, nous croyons que la faible industrialisation de Québec et l'attractivité de Montréal au cours du XXe siècle constituent les principaux facteurs explicatifs.

\begin{tabular}{|l|c|c|c|c|c|c|}
\hline & $\begin{array}{c}\text { Population } \\
\mathbf{2 0 1 6}\end{array}$ & $\begin{array}{c}\text { Variation } \\
\mathbf{2 0 1 1 / 2 0 1 1 6}\end{array}$ & $\begin{array}{c}\text { \% pop. } \\
\text { Ville-RMR }\end{array}$ & $\begin{array}{c}\text { Densité 2016 } \\
\text { (hab. par km²) }\end{array}$ & $\begin{array}{c}\text { \% de non- } \\
\text { immigrants }\end{array}$ & $\begin{array}{c}\text { Taux d'inoccu- } \\
\text { pation } \\
\text { (logements 2017) }\end{array}$ \\
\hline Montréal & 4098927 & $4,2 \%$ & $41,6 \%$ & 890,2 & $74,9 \%$ & 2,8 \\
\hline Ottawa-Gatineau & 1323783 & $5,5 \%$ & $70,6 \%$ & 195,6 & $79,0 \%$ & 1,7 \\
\hline Québec & 800296 & $\mathbf{4 , 3} \%$ & $\mathbf{6 6 , 5} \%$ & $\mathbf{2 3 4 , 8}$ & $\mathbf{9 3 , 6} \%$ & $\mathbf{4 , 5}$ \\
\hline Winnipeg & 778489 & $6,6 \%$ & $90,6 \%$ & 146,7 & $74,3 \%$ & 2,8 \\
\hline Hamilton & 747545 & $3,7 \%$ & $71,8 \%$ & 744,9 & $74,9 \%$ & 2,4 \\
\hline
\end{tabular}

Tableau 1 - Comparaison de cinq RMR canadiennes (populations, densités et logements) Source : Statistique Canada, 2011 et 2016; SCHL, 2018

En matière d'économie, Québec a un taux de chômage plus faible que Montréal ou que la moyenne provinciale, c'est-à-dire 4,6\% (RMR de Québec), contre 7,5\% (RMR de Montréal), toujours selon les données du recensement de 2016 (Statistique Canada, 2016c). Néanmoins, en 2017, le taux d'inoccupation 
des logements de la capitale est de 4,5\%, contre 2,8 \% pour la métropole (SCHL, 2018). Pour relativiser cette information, on peut dire que le taux d'inoccupation est une donnée assez volatile qui peut augmenter après quelques années de fortes mises en chantier dans le secteur résidentiel d'une région métropolitaine, et vice-versa. Au demeurant, Québec s'enorgueillit d'un nouvel amphithéâtre sportif depuis 2015 et de l'arrivée du géant commercial IKEA en 2018.

Cette ère de croissance coïncide en grande partie avec le règne du maire Régis Labeaume, un politicien au franc-parler en poste depuis 2007. Il s'agit du maire ayant le plus long règne depuis le regroupement municipal de 2002, qui a réuni 11 anciennes municipalités locales, comme ce fut le cas à Montréal et dans d'autres centres urbains du Québec ${ }^{6}$. À Québec, cette fusion de la ville centre et des villes de banlieue est un événement historique qui fait suite à des décennies de tensions intermunicipales ayant eu lieu au sein de la défunte Communauté urbaine de Québec (Hulbert, 1994). Perçu comme un leader fort porté sur l'action, Labeaume jouit d'une grande popularité auprès de la population et il est à l'avant-scène de la vie municipale (Belley, Quesnel et Villeneuve, 2011). Pour certains, son étoile commence à pâlir à cause de ses tendances autoritaires (Porter, 2018c).

\section{Les dilemmes urbanistiques actuels}

C'est dans ce contexte qu'ont émergé les grands projets urbanistiques à l'ordre du jour. Véritables travaux d'Hercule, ces projets sont ambitieux et soulèvent différents enjeux pratiques et conceptuels en matière d'urbanisme. Deux de ceux-ci sont des projets publics : le réseau de tramway et le $3 e$ lien entre les rives du fleuve Saint-Laurent, alors que le projet du Phare est de nature privée (voir figure 3). Commençons par discuter du projet de réseau de tramway, un projet relativement ancien qui est revenu à l'agenda en 2017.

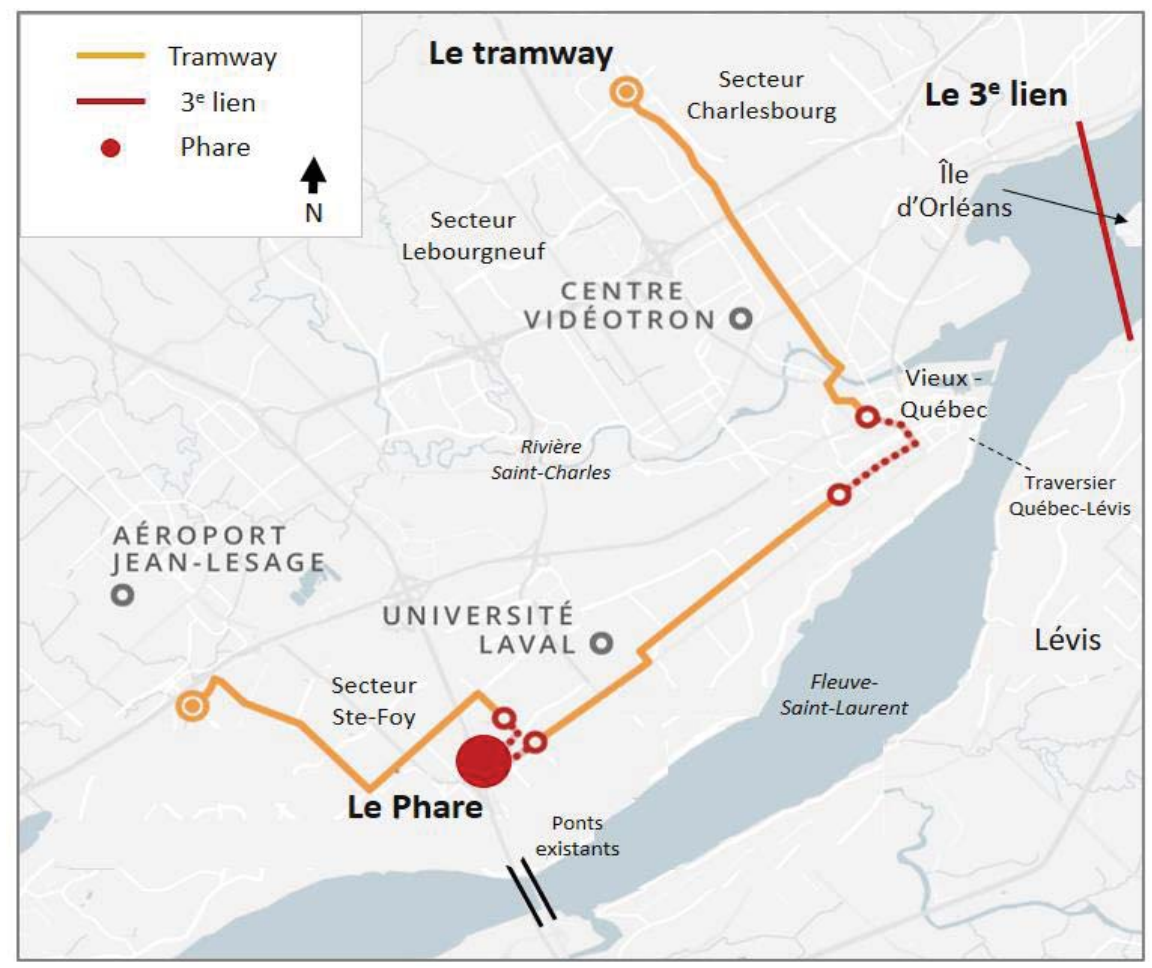

Figure 3 - Les trois dilemmes urbanistiques de Québec Source : SRC, 2018; modifiée par Martin Simard, 2019

\subsection{Le projet de tramway à Québec}

Comme le dénoncent les militants en faveur du développement durable, Québec est la seule grande ville canadienne à ne pas avoir de système de transport collectif de type métro ou tramway (Lavallée,
2017). Il y a bien un système de transport en commun par autobus, mais celui-ci serait moins efficace ou structurant qu'un système de transport hors route, malgré l'existence du métrobus et de voies réservées aux heures de pointe depuis 1992. Il con- 
vient de rappeler que Québec a déjà eu des tramways sur ses routes entre 1897 et 1948 (Porter, 2018a). Comme dans la plupart des villes nordaméricaines, ceux-ci ont disparu dans les années 1940 ou 1950 pour diverses raisons : faible qualité du service, baisse de la clientèle, compétition de l'automobile et de l'autobus, etc. (Faugier, 2009).

Depuis environ 20 ans, le tramway revient à la mode. Celui-ci est aujourd'hui vu comme une forme de mobilité durable, en plus d'être un équipement moderne et esthétique qui favorise la réhabilitation des espaces urbains centraux (Lois González, Pazos Otón et Wolff, 2013). Moins massifs et plus silencieux, les tramways de notre époque s'intègreraient mieux à la trame urbaine que leurs ancêtres et même mieux que les autobus actuellement en usage. Le tramway est aussi beaucoup moins cher à mettre en place qu'un métro souterrain et il peut fonctionner sur le réseau routier existant aussi bien que sur des emprises qui lui sont dédiées, selon l'expression consacrée. Plusieurs grandes villes françaises ou américaines se sont récemment dotées de réseau de tramway, notamment Bordeaux et Strasbourg, ou encore Minneapolis (Minnesota) et Portland (Oregon).

À Québec, l'idée du retour du tramway date du début des années 2000, malgré des mentions occasionnelles dans des rapports d'experts au cours des décennies précédentes (Ville de Québec, 2011). Le Réseau de transport de la Capitale (RTC), organisme responsable du transport en commun, produit alors une étude sur le sujet à la suite d'une demande du ministère des Transports de la province. À la lumière des exemples provenant de différentes villes, le rapport conclut ceci : «La mise en place du tramway [aurait] des effets bénéfiques sur le développement urbain, l'aménagement du territoire, l'image du transport en commun et son achalandage » (RTC, 2003, p. 5). De plus, il n'y aurait pas de problèmes techniques particuliers liés à la réalisation de ce projet et les coûts ne seraient pas prohibitifs, toujours selon le RTC (voir figure 4).

Le projet est accueilli très favorablement par le maire de l'époque, Jean-Paul L'Allier. Toutefois, en ces années intenses de gestion administrative et politique du regroupement municipal, le projet ne sera pas lancé (Béland, 2017). En 2005, la nouvelle mairesse issue de la banlieue, Andrée Boucher, se montrera réticente au projet, qu'elle qualifie de " rêve » peu avant son décès (Porter, 2018a). Son successeur en 2007, Régis Labeaume, tiendra des propos ambigus par rapport au tramway au cours des 12 années suivantes, appuyant et rejetant en alternance l'idée, notamment pour des questions de financement et à la suite des désaccords sur les tracés possibles.

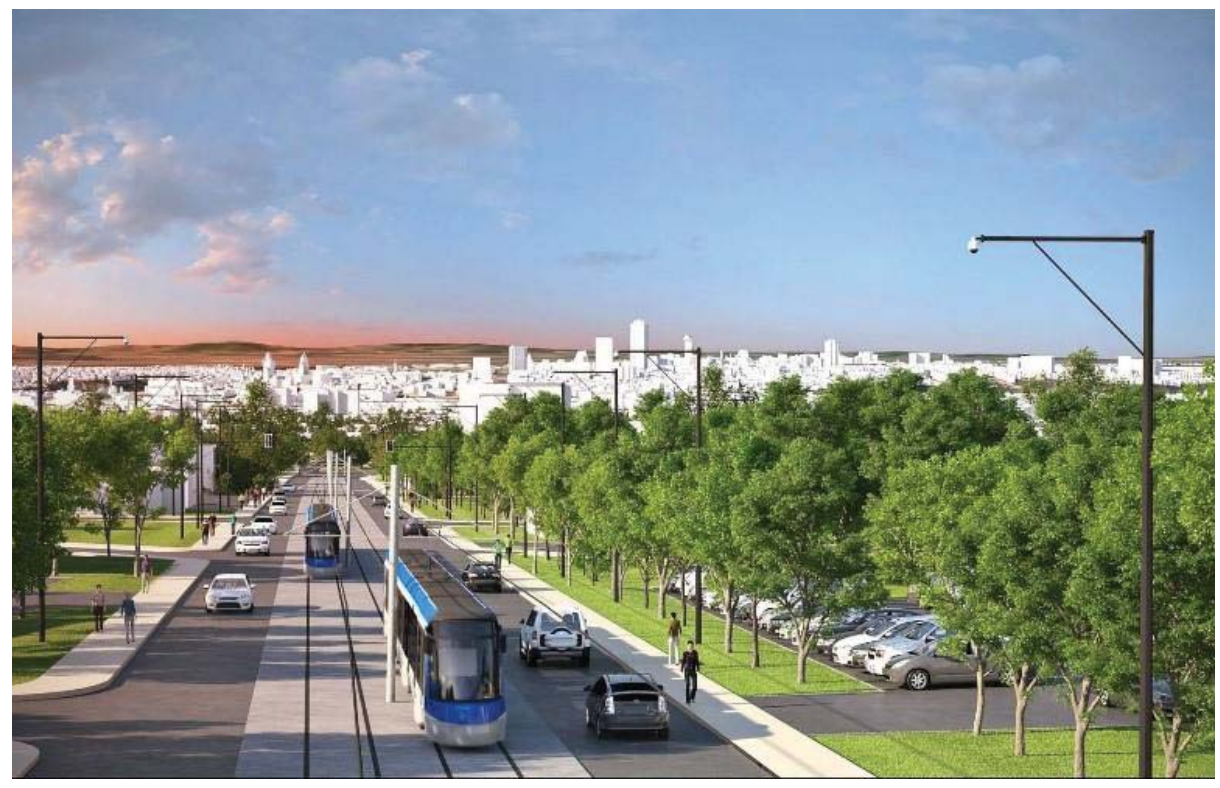

Figure 4 - Simulation d'un tramway dans le secteur de Charlesbourg Source : Ville de Québec, 2019 
D'abord favorable au concept, le maire Labeaume entérinera un projet de tramway par l'adoption du Plan de mobilité durable de la ville (2011) et par l'entremise du mandat attribué au RTC de réaliser une étude technique de faisabilité. Pourtant, sans attendre les conclusions de cette étude, le maire affirme qu'il priorise désormais l'automobile et la lutte à la congestion routière (Morin, 2013). En 2015, nouveau revirement: Régis Labeaume défend plutôt l'implantation d'une infrastructure de service rapide par bus (SRB) en collaboration avec la Ville de Lévis, sa voisine sur la rive sud du fleuve qui fait partie de la RMR de Québec (Morin et Gaudreau, 2015). Après de nombreuses tergiversations, les relations entre les deux villes voisines se corsent et le projet de SRB est abandonné en avril 2017 (Porter, 2018a).

Quelques mois plus tard, le maire Labeaume revient à la charge et annonce officiellement, en compagnie du premier ministre québécois de l'époque, M. Philippe Couillard, un projet de 3,3 G\$ et de $56 \mathrm{~km}$, qui est qualifié de Réseau structurant de transport en commun, lequel projet sera restreint à la rive nord de l'agglomération (Boisvert, 2018a). Le projet renouvelé est appuyé par plusieurs organismes, dont le Club automobile du Québec (CAA) et l'Institut du développement urbain (IDU). Le nouveau premier ministre du Québec, M. François Legault, réitère l'appui du gouvernement au projet à la suite de son élection en octobre 2018 (Moalla, 2018a). Le dossier est présenté par le maire de Québec comme étant urgent et le premier magistrat de la capitale exige que l'habituelle étape du Bureau d'audiences publiques sur l'environnement $(\mathrm{BAPE})^{7}$ soit évitée ou écourtée (Porter, 2019; Moalla, 2019).

Au peintemps 2019, les journaux parlent quotidiennement du conflit fédéral-provincial sur le financement. De plus, l'annonce des détails du projet ${ }^{8}$ soulève certaines controverses, comme celle de la desserte ou non du complexe immobilier Marly de Revenu Québec ou l'éventuelle compensation des commerçants lors des travaux à venir. Finalement, le projet est officiellement lancé le 19 août 2019 par le premier ministre Trudeau, à la suite d'une entente formelle entre les gouvernements sur les modalités de financement du projet.

\subsection{Le projet de $3^{\mathrm{e}}$ lien routier entre Québec et Lévis}

Même si elle est autonome sur le plan politique et qu'elle est incluse dans une autre région administrative, la Ville de Lévis est fermement intégrée à la RMR de Québec. Ce phénomène s'est développé progressivement au cours des 60 dernières années. Ainsi, la Ville de Lévis peut être considérée comme une banlieue de Québec, malgré son ancienneté et ses fonctions centrales. De plus, les divers pôles d'emplois de la rive sud stimulent également les échanges entre les rives du fleuve Saint-Laurent ${ }^{9}$.

Ces échanges sont supportés par deux ponts dans le secteur ouest de l'agglomération, soit le pont de Québec, de type cantilever, qui a été mis en service en 1919 après moult péripéties (L’Hébreux, 2008), et le pont Pierre-Laporte (voir figure 5), un pont suspendu de plus grande capacité ouvert à la circulation en 1970 (Dupuis, 2013). Au cœur de l'agglomération, on remarque aussi un traversier entre le Vieux-Québec et Lévis, mais il s'agit d'un système d'appoint multimodal au débit relativement limité.

Les données généralement utilisées sur la circulation interrives dans la RMR de Québec proviennent du rapport de l'enquête Origine-Destination 2011 (MTQ et collab., 2015, cité par Fortin, 2016) ${ }^{10}$. Celles-ci indiquent qu'il y a 15200 passages par jour sur les deux ponts entre Québec et Lévis. La grande majorité de ces déplacements sont effectués par des automobilistes habitant la rive sud. Par ailleurs, $76 \%$ des navetteurs vers Québec proviennent de l'ouest de Lévis ou des municipalités voisines, tandis que $66 \%$ des navetteurs provenant de la rive sud ont une destination située dans l'ouest de la rive nord de l'agglomération de Québec.

Ces données permettent de saisir l'intensité du trafic interrives, la nécessité ou non d'un $3^{e}$ lien ainsi que les patrons de déplacements, lesquels fournissent accessoirement des pistes pour la localisation d'un éventuel $3^{e}$ lien. Les informations sorties récemment confirment les trajectoires des déplacements véhiculaires, lesquels se concentrent dans l'ouest de l'agglomération. 


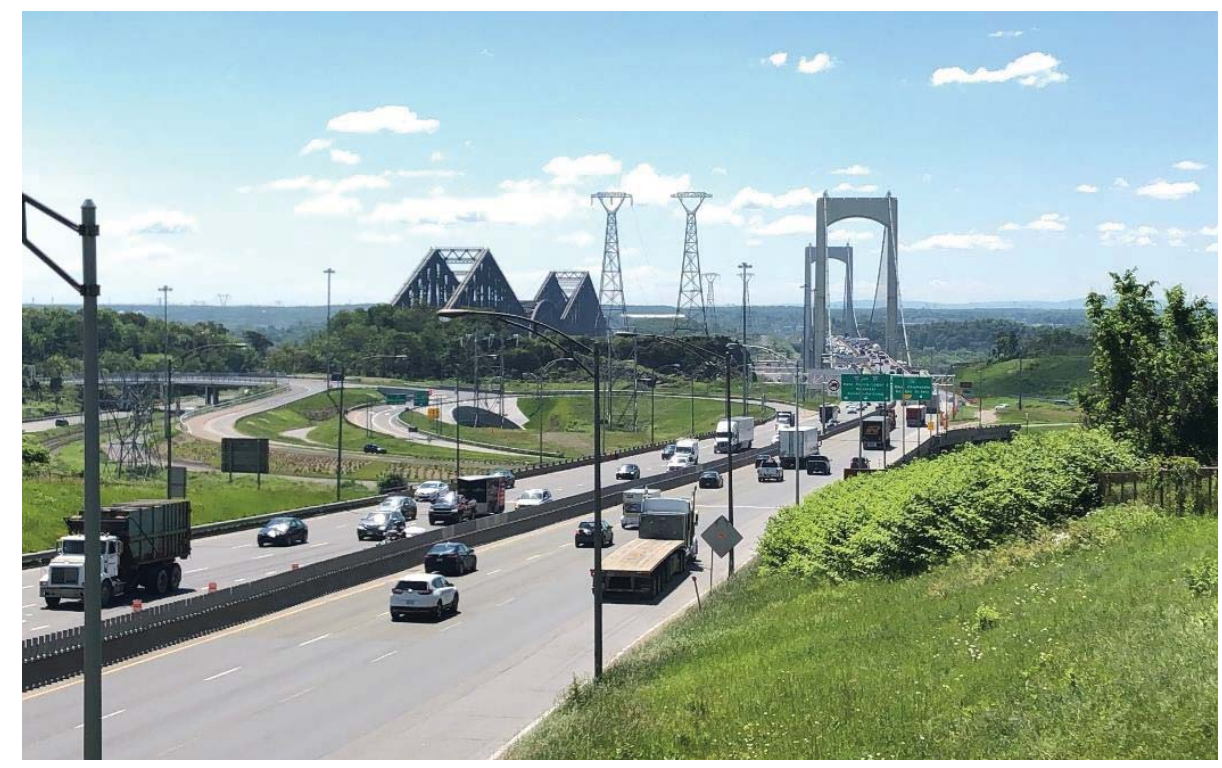

Figure 5 - Les ponts de Québec et Pierre-Laporte (vue du nord vers le sud) Source : Martin Simard, 2019

L'idée d'un 3e lien interrives à Québec circule depuis près de 15 ans. Elle est notamment discutée abondamment sur les ondes des stations de radio régionales. Cet intérêt découle de l'augmentation notable de la circulation routière à Québec depuis le début des années 2000 (Pelchat, 2009). Il faut garder en mémoire le fait que Québec présente un fort taux de motorisation. De plus, selon Statistique Canada (2016b), 80,4\% de la population active de la RMR de Québec utilisait l'automobile, un camion léger ou une fourgonnette pour ses déplacements quotidiens, en 201611. La Coalition avenir Québec (CAQ) prend position en faveur du projet de $3^{\mathrm{e}}$ lien lors de l'élection provinciale de 2014 et le Parti libéral du Québec en fait autant en 2017, en plus de procéder à la mise en place d'un «Bureau de projet » pour évaluer la faisabilité des multiples options d'aménagement (Borde, 2017).

En août 2018, le fameux Bureau fera connaitre les résultats de ses études en pleine campagne électorale : cinq trajets potentiels sont proposés au coût de plusieurs milliards de dollars, avec une phase de construction planifiée pour débuter en 2026 (Gagnon, 2018). Le maire de Québec a déjà fait connaittre sa préférence pour un corridor possible à l'ouest des ponts actuels (Morin, 2016). D'autres acteurs favorisent plutôt les corridors éventuellement situés à l'est de l'agglomération, notamment le nouveau gouvernement élu à Québec en octobre 2018, et ce, malgré les effets potentiels sur le paysage (Bourque, 2018a). L'avenir du pont reliant la rive nord du fleuve à l'île
d'Orléans est aussi mis dans la balance. C'est alors que la vieille idée d'un tunnel sous le fleuve Saint-Laurent revient à l'avant-scène, avec ou sans raccordement avec l'île. Par ailleurs, le gouvernement de la CAQ espère aussi entamer rapidement la construction du projet, soit dès 2022 .

Pour les opposants au projet, c'est le concept même de $3^{e}$ lien qu'il faut remettre en question dans une optique de lutte à l'étalement urbain et de développement durable (Grandmont, 2018). Le lien interrives est aussi vu comme contraire au projet de tramway, ce dernier visant la diminution de la circulation automobile et la densification urbaine, projet qu'on souhaite pourtant réaliser en parallèle au $3^{\mathrm{e}}$ lien (Simard, 2018). En référence au probable nouvel élan du développement urbain sur la rive sud et à l'appétit présumé des promoteurs immobiliers envers les opportunités d'affaires à venir en relation avec le nouveau lien de transport, un député du parti Québec solidaire assimile celui-ci à l'attrait d'une « ligne de coke » pour les adeptes des drogues (Gagné, 2018a). Cette affirmation-choc ne sera pas la dernière au sujet de ce grand projet d'infrastructure très controversé, qui est toujours à l'agenda au mi-temps de l'année 2019.

\subsection{Le projet immobilier du Phare de Québec}

Un troisième projet d'envergure est au cœur des débats publics dans la RMR de Québec. Il s'agit cette fois d'un projet immobilier privé, soit le complexe Le 
Phare. Si l'on ne traite plus ici d'un projet instauré par les instances publiques, les autorités municipales ont malgré tout un rôle primordial à jouer en matière d'évaluation, de négociation des modalités et d'autorisation finale de cet imposant projet.

Sans doute inspiré par l'édifice du Burj Khalifa à Dubaï, le promoteur Michel Dallaire, président du
Fonds de placement immobilier Cominar, veut construire le plus haut édifice canadien à l'est de Toronto, soit un ensemble comprenant des commerces, des bureaux et des logements répartis sur trois tours, dont la principale devrait atteindre 65 étages d'une hauteur de $250 \mathrm{~m}$. Le projet, perçu par certains comme démesuré, serait localisé dans le secteur de Sainte-Foy, près des accès aux ponts de Québec et Pierre-Laporte (voir figure 6).



Figure 6 - Esquisse du projet Le Phare de Québec Source : Groupe Dallaire, 2018

L'intention de construire Le Phare a été dévoilée en janvier 2015 et le projet a immédiatement suscité des critiques : «L'intervention ne se rattache ainsi à rien, sinon à des rêves de grandeur. [...] On se retrouve ainsi, à Québec, avec un projet sans lien avec son environnement, en rupture d'échelle, qui n'a d'audace que la hauteur » (Cardinal, 2015). L'entreprise de plus de $750 \mathrm{M} \$$ parait invraisemblable dans une agglomération de moins d'un million d'habitants, ville patrimoniale qui ne dispose pas d'un centre d'affaires à l'américaine (Demers et collab., 2015). Le projet est aussi décrit comme tirant sa source dans le « complexe d'infériorité de Québec » par rapport à Montréal (Robitaille, 2015).

Plusieurs interrogations pratiques balisent également les commentaires des acteurs locaux et régionaux (Bourque, 2015) : Est-ce que les marchés de l'espace commercial et du logement de type condominium pourront absorber un tel afflux de nouveaux espaces $\left(270000 \mathrm{~m}^{2}\right.$ de bureaux et commerces ainsi que 1000 nouveaux logements)? La localisation du complexe en banlieue risque-t-elle de "décentrer» Québec? Le site choisi, situé à proximité des ponts, générera-t-il un trafic considérable dans un secteur déjà congestionné? C'est sans compter les effets de voisinage (ombrage, couloirs de vents, impression visuelle d'écrasement sur les bâtiments limitrophes, évaluation foncière à la hausse et mouvements prévisibles de spéculation foncière, etc.).

Dès 2011, une stratégie de densification du secteur du boulevard Laurier à Sainte-Foy était mise de l'avant pour ce pôle commercial et de bureaux qui rivalise avec le centre-ville de l'agglomération depuis quatre décennies (Mercier et Roy, 2014). Cette volonté a été inscrite dans le Programme particulier d'urbanisme (PPU) du Plateau centre de Sainte-Foy, l'année suivante, malgré les réticences de plusieurs citoyens. Néanmoins, la taille maximale autorisée dans le document officiel est de 29 étages (Ville de Québec, 2012). Le projet du Phare dépasse donc largement la réglementation en vigueur. Une modification au PPU aurait dû donner lieu à un référendum des résidents 
voisins du projet, mais la municipalité a plutôt fait appel à l'article 74.4 de la Charte de la Ville de Québec ${ }^{12}$, qui lui permet de modifier sa réglementation de zonage sans référendum (Bourque, 2018b).

Néanmoins, la Ville de Québec a organisé des séances d'information, les 29 et 30 octobre 2018, et une soirée de consultation publique, le 21 novembre suivant ${ }^{13}$. Celles-ci ont été fort courues et les commentaires des participants étaient « très contrastés » (Moalla, 2018b), voire majoritairement défavorables (Gagné, 2018b). Au-delà des informations fournies, plusieurs participants ont eu l'impression que la décision d'aller de l'avant avec le projet était déjà prise. Un observateur de la scène municipale soutiendra que « le bulldozer du Phare est en marche et rien ne semble plus pouvoir le freiner» (Bourque, 2018c). Le maire était absent lors des séances d'information et de consultation, mais il a minimisé le rôle de celles-ci dans une déclaration médiatique (St-Onge, 2018). On prévoit lancer le chantier du Phare à l'automne 2019 ou au printemps 2020.

\section{Les enseignements du cheminement des projets urbains}

Les trois grands projets à l'étude ici interpellent non seulement les citoyens sensibles à la qualité de leur milieu de vie, mais également certains principes théoriques à la base de l'urbanisme. En effet, ce qu'on nomme la théorie de la planification a fait l'objet d'échanges innombrables depuis les années 1960, notamment dans la littérature scientifique anglosaxonne (Fainstein et Defilippis, 2016). En France, on remarque un intérêt nouveau envers ce sujet depuis une décennie (Douay, 2013; Demazière, 2015; Debrie, 2018). Les enjeux généralement traités à l'intérieur de ces écrits réfèrent au processus de prise de décision, aux rôles respectifs des élus, des experts et des citoyens ainsi qu'aux concepts d'intérêt collectif et de bien commun (Baché et Gauthier, 2011). Plus récemment, la question de la durabilité est entrée en scène pour interroger les modalités et la finalité des projets urbains (Roseland, 2012; Roudil et Roudil, 2012).

Il serait long et ardu de résumer toutes les discussions sur la théorie de la planification. Cependant, le texte relativement récent de Fischler (2015) a tenté l'expérience avec un certain succès. Celui-ci se veut d'ailleurs critique de la discipline : «L'urbanisme est à la fois mis au défi et enrichi par la présence au cœur de son identité d'un certain nombre de contradictions et de tensions » (Fischler, 2015, s. p.). Malgré ces contradictions et tensions, plusieurs principes de base obtiennent une adhésion très large auprès des analystes et des professionnels. Dans ce contexte, nous utiliserons quelques thèses identifiées par Fischler pour évaluer les processus de planification et de décision ayant été employés pour les grands projets de Québec.

\subsection{Le caractère politique de l'urbanisme}

Comme le dit le professeur-urbaniste œuvrant à l'Université McGill, «l'urbanisme est une activité politique [thèse 12] et [il] est façonné par des valeurs, c'est-à-dire par des conceptions de la manière [...] dont l'environnement doit être agencé pour que l'on vive bien [en] société [thèse 13]» (Fischler, 2015, s. p.). Il ajoute que «les urbanistes ne sont pas des acteurs très puissants dans le processus de planification urbaine. Les élus et les investisseurs sont les joueurs les plus puissants [thèse 39]». La prise de décision relèverait conséquemment des élus politiques, tandis que les urbanistes fourniraient des analyses et recommandations appuyées par des chiffres et des avis basés sur l'expérience accumulée dans ce champ d'activité institutionnalisé depuis près d'un siècle en Occident.

Si l'on applique ces principes aux trois projets à l'étude, on réalise que le rôle des élus est prépondérant à Québec et que ceux-ci décident en fonction des valeurs et/ou des programmes diffusés avant leur élection. C'est particulièrement le cas pour nos exemples parce que deux des trois projets concernent les infrastructures de transport, lesquelles sont habituellement de responsabilité publique. Ce rôle actif n'est pas un mal en soi, car l'essence du politique est de faire des choix.

Cependant, les projets d'aménagement supportés par les élus n'ont pas tous été discutés lors des campagnes électorales. Dans le cas du complexe immobilier Le Phare, ce projet du secteur privé n'a pas été abordé lors de l'élection municipale de 2013 à Québec. En outre, il contrevient au PPU du Plateau centre de Sainte-Foy, adopté en 2012 par la même administration après une vaste consultation (Boisvert, 2018b). L'enjeu de la densité était alors au centre des discussions. 
L'urbanisme est une pratique fondamentalement gérée à l'échelle locale ou microrégionale. Toutefois, pour certains projets d'infrastructures ou touchant des terres fédérales ou provinciales, on doit composer avec plusieurs paliers de gouvernement (fédéral, provincial et municipal). Ceux-ci tentent d'harmoniser leurs actions dans un contexte de hiérarchie des pouvoirs et des moyens, et à travers la diversité des idéologies et des points de vue. Cela se fait avec plus ou moins de succès.

Au-delà de l'opinion des citoyens, la mise en œuvre de projets complexes nécessite donc un rarissime alignement de planètes. Dans ce contexte, le projet de 3e lien a clairement été mentionné dans son programme par la CAQ avant que le parti ne prenne les commandes du gouvernement québécois. Son appui au projet de réseau de tramway a été plus timide, contrairement au gouvernement fédéral, qui avait précédemment promis du financement au maire de Québec, ce qui a relancé ce dernier projet. Au palier municipal, l'appui au projet de $3^{e}$ lien a été tardif et, en quelque sorte, forcé par l'arrivée au pouvoir de la CAQ (Bourque, 2018a).

Bref, deux des trois projets à l'étude ont été imposés ou fortement encouragés par les gouvernements supérieurs, qui n’ont théoriquement pas compétence en matière d'aménagement et d'urbanisme. Ce manque de respect des prérogatives des instances politiques décentralisées apparaît condamnable. Néanmoins, cela ne veut pas dire que la population est en désaccord avec ces projets structurants ou que ces derniers sont illégitimes, si l'on considère qu'ils ont fait l'objet de " promesses électorales».

Dans le dossier du Phare, une intervention urbanistique plus classique issue du secteur privé, les paliers fédéral et provincial n'ont pas eu à se prononcer ou n'ont pas voulu le faire. Le palier local a poussé le dossier avec vigueur, en dépit des réticences émises lors des consultations formelles ayant été effectuées. Dans ce cas précis, la légitimité de l'administration municipale est plus ténue, car le projet est à l'opposé des plans et règlements adoptés quelques années plus tôt pour le secteur de Sainte-Foy.

\subsection{L'idéal de la participation et des consultations publiques}

Il convient maintenant de présenter deux autres principes énoncés par Fischler : «Un urbanisme de qualité en termes de processus [est] une opportunité d'apprentissage collectif et de délibération collective [thèse 23] ». D'autre part, «les bons plans bénéficient de l'apport de toutes les personnes et institutions dont la collaboration est requise pour leur mise en œuvre [thèse 27] » (Fischler, 2015, s. p.). Ces règles proposées réfèrent à la question de la participation de divers groupes d'acteurs, notamment des citoyens. Les consultations publiques en matière d'urbanisme se sont généralisées depuis environ 50 ans, mais leur application est fort inégale sur les plans quantitatif et qualitatif (Baché et Gauthier, 2011). De surcroit, même les démarches les plus élargies et les mieux intentionnées ne sont pas garantes de l'atteinte de consensus dans nos sociétés pluralistes, malgré les aspects positifs de l'échange d'arguments et d'informations (Simard, 2003).

Un regard sur nos trois projets à la lumière de ces principes nous permet de constater que la participation des citoyens diffère d'un projet à l'autre, mais que celle-ci n'a été exemplaire dans aucun des dossiers majeurs touchant la région métropolitaine de Québec.

En premier lieu, le concept du $3^{e}$ lien routier interrives n'a fait l'objet d'aucune délibération collective organisée. Le projet a été moussé dans le monde médiatique pour être rattrapé par électoralisme par la CAQ, parti politique qui allait éventuellement détenir le pouvoir. Lidée qui semble jouir d'un large appui, en particulier dans les banlieues des deux rives de l'agglomération, parait être le résultat d'un «construit social» imperméable à toute discussion. Sa nécessité irait de soi chez les citoyens empruntant quotidiennement les routes congestionnées de la RMR, aux heures de pointe. Pour leur part, les militants en faveur de l'environnement et d'une ville durable s'opposent au projet, mais leurs arguments sont souvent jugés comme étant de nature «idéologique ». Cette polarisation des valeurs et des représentations laisse peu de place à la discussion.

La situation est plus complexe pour les deux autres projets. Le réseau de tramway a suscité des discussions collectives à diverses reprises, mais les changements répétés apportés au projet et les longs délais ont gommé les effets d'une démarche participative autrement assez étendue. Plus précisément, il y a eu des débats sur ce sujet lors des consultations sur le Plan de mobilité durable de la Communauté métropolitaine de Québec en 2009 et 2010, pendant les consultations sur le Plan métropolitain d'aménagement et de 
développement (PMAD) en 2011 et au cours de l'élaboration du Programme particulier d'urbanisme (PPU) du Plateau centre de Sainte-Foy en 2012. Un comité consultatif avait aussi été créé pour étudier la question à la fin des années 2000. La question du tracé avait créé des dissensions au sein du groupe et les recommandations étaient restées lettre morte pour diverses causes, en particulier la dimension financière du projet. Le projet remodelé mis sur la table en 2017 a été suivi de consultations publiques les 4, 5, 7 et 11 avril 2018 et de séances d'information sur le tracé et les stations les 11, 13 et 18 juin 2019 (voir figure 7) ${ }^{14}$.

En ce qui concerne Le Phare, nous avons déjà souligné la tenue de trois soirées d'information ou de consultation sur le sujet. Toutefois, celles-ci ont été largement discréditées par les commentaires du maire Labeaume ayant mis en cause la représentativité des participants (St-Onge, 2018). Une conseillère municipale a également affirmé que les différents projets d'urbanisme ne peuvent pas plaire à tous et que l'administration municipale doit statuer sur la base de l'intérêt de l'ensemble de la collectivité, et non seulement des gens vivant à proximité du projet (Gagné, 2018b). On doit ici se remémorer que la municipalité avait usé de stratégie pour que la modification au règlement de zonage ne soit pas soumise à un référendum auprès des résidents de la zone limitrophe, contrairement à ce qui se fait régulièrement au Québec (Bourque, 2018b) ${ }^{15}$.

\subsection{L'importance des études pour supporter les interventions}

Revenons aux thèses développées par Raphaël Fischler. Celui-ci rappelle que «la planification urbaine est un moyen de rationaliser le processus par lequel [les acteurs] utilisent le sol et d'autres ressources pour subvenir à leurs besoins [thèse 18] ». Toujours selon le même auteur, « les urbanistes sont censés être objectifs dans l'analyse des faits [...] et rationnels dans l'élaboration de recommandations [thèse 33]» (Fischler, 2015, s. p.). En somme, l'urbanisme aspire à enrichir le processus politique local à partir d'informations, de données et d'analyses plus éloignées des considérations politiques et stratégiques qui guident généralement les politiciens.

À ce chapitre, les cas observés laissent transparaitre que les idées et concepts promus par les différents paliers gouvernementaux en matière d'urbanisme ne sont pas toujours appuyés par des études de faisabilité ou d'impact. Cela est particulièrement visible en ce qui concerne le projet du 3e lien entre Québec et Lévis, dont la nécessité reste à prouver (La Presse canadienne, 2019), sans compter les difficultés techniques et les coûts très élevés qui sont prévisibles. Les chiffres peuvent même être manipulés de manière à justifier un projet. Le ministre des Transports François Bonnardel l'a éloquemment démontré en utilisant de manière inappropriée les données de l'enquête Origine-Destination dévoilée en mai 2019 (Néron, 2019).

Dans la même veine, les enjeux urbanistiques sont souvent traités comme des questions d'opinion. À l'ère du relativisme, on peut être pour ou contre, sans avoir à étayer précisément sa position. De surcroit, l'intérêt pour les délibérations publiques n'est pas toujours présent. En conséquence, un parti politique propose une intervention et on vote en fonction de celle-ci; le reste ne serait que détails techniques à reléguer aux technocrates ou aux experts en tout genre.

Or, il s'avère que les dilemmes urbanistiques existant à Québec comportent des dimensions techniques importantes qui commandent une expertise ne se limitant pas à l'urbanisme, mais comportant des dimensions architecturales et, surtout, d'ingénierie. Ces aspects techniques soulevant peu d'attention (p. ex., dans le cas du $3^{e}$ lien) devraient normalement faire la différence entre une acceptation ou un refus du projet. Qu'à cela ne tienne, les arguments qui contredisent les positions des acteurs locaux ou des individus sont perçus comme des informations fallacieuses (fake news) ou des légendes urbaines. Pourquoi le monde ne pourrait-il pas prendre la forme que l'humain souhaite lui donner? On pousse ici à l'extrême la croyance moderniste qu'on peut façonner la nature selon nos bons désirs, à une époque où le développement durable devrait pourtant nous mener dans une direction contraire.

\subsection{Les capacités prévisionnelles de l'urbanisme de planification}

Pour une dernière fois, référons aux thèses de Fischler : «Comme la plupart des gens, les urbanistes ont trop souvent tendance à ignorer le fait que les villes sont des systèmes complexes $[\ldots]$ que les urbanistes ne contrôlent que de manière minimale [thèse 37]. » Dans la même veine, « la recherche universitaire n'a qu'une valeur limitée pour la pratique de 
l'urbanisme. [Toutefois,] les professionnels peuvent apprendre grâce à elle ce qui semble marcher dans telle ou telle circonstance, quels facteurs semblent être critiques à la réussite, et quels problèmes ils peuvent anticiper [thèse 49] » (Fischler, 2015, s. p.). Cette autocritique des méthodes et possibilités de l'urbanisme nous fait réaliser qu'il ne s'agit pas d'une science exacte. Doit-on pour autant mettre de côté les recherches et prévisions des planificateurs urbains?

Ce débat fondamental a été mis en branle par les propos d'un citoyen dans un quotidien de la région de Québec. Celui-ci, de toute évidence partisan du $3^{e}$ lien entre les deux rives, dénonçait les inquiétudes sur le plan de l'étalement urbain, inquiétudes répétées par les opposants au projet et par les universitaires du milieu, acteurs qui prônent davantage une ville compacte et le développement durable de l'agglomération. Le citoyen affirmait que «les conditions de laboratoire n'existent pas en urbanisme" (Verville, 2018, s. p.). Cet énoncé est vrai. Néanmoins, doit-on en conclure que l'expertise des urbanistes est limitée, voire nulle? En réalité, l'urbanisme s'alimente aux leçons des expériences passées. Dans cette optique, les éléphants blancs et les projets bétonnés des années 1960 et 1970 sont là pour nous inciter à la prudence. D'ailleurs, à Québec même, les urbanistes travaillent depuis plus de 20 ans à corriger les erreurs du passé (Mercier, 2000).

Si l'on revient à la question des prévisions, même si le contexte de fonctionnement du système urbain local n'est pas le même partout, les expériences nordaméricaines indiquent qu'une augmentation de l'offre en transport automobile, dans une agglomération en croissance, produit de l'étalement urbain et ramène, à moyen terme, la congestion routière sur les nouveaux tronçons autoroutiers. Pourquoi se priver de cet élément de connaissance qui s'est avéré véridique à de multiples occasions? Évidemment, le phénomène risque de se produire ici à une intensité et à un rythme particuliers, mais son avènement est une quasicertitude. D'ailleurs, il est fort à parier que des promoteurs immobiliers et des spéculateurs s'activent déjà dans le secteur sud-est de la RMR de Québec en vue de capter la plus-value foncière qui résultera d'une forte amélioration de l'accessibilité du secteur, avec l'avènement du $3 \mathrm{e}$ lien.

\section{Conclusion}

La région métropolitaine de Québec fait face actuellement à trois grands dilemmes urbanistiques. Par leur ampleur et leur caractère structurant (ou déstructurant), ces projets urbains affecteront l'organisation spatiale et le paysage de l'agglomération pour plusieurs générations. Ces projets coûteux obligeant la coordination de multiples paliers de gouvernement et du secteur privé sont complexes sur le plan technique, et leur réalisation se fera sur plusieurs années, voire plus d'une décennie. L'importance de réfléchir et de délibérer afin de prendre les bonnes décisions s'impose, mais ce n'est pas exactement ce qui se produit sur le terrain à Québec, comme c'est probablement souvent le cas ailleurs.

Si l'on étudie les interventions projetées dans une perspective conceptuelle ou théorique dans le champ de l'urbanisme, plusieurs enseignements peuvent en ressortir.

Premièrement, ces projets sont promus par des leaders politiques qui en font une affaire personnelle, dans bien des cas. En conséquence, ces idées sont défendues avec un certain autoritarisme. On cherche aussi à les mettre en œuvre rapidement, soit selon la temporalité des échéances électorales. Finalement, les débats sur les actions proposées sont fréquemment empreints de partisanerie. D'ailleurs, le mandat issu du système de démocratie de représentation est mis de l'avant pour légitimer l'avis des élus par rapport aux commentaires des citoyens ou des positions des groupes organisés, par exemple lors des consultations publiques. L'arrimage de la démocratie de représentation et de mesures issues de la démocratie directe trouverait ici ses limites.

En second lieu, la participation des citoyens est souvent assurée. La Ville de Québec possède une Politique de participation publique ${ }^{16}$. Les séances sont annoncées à l'avance et font l'objet de rapports formels, même d'enregistrements intégraux accessibles au public. Cependant, dans le cas des projets à l'étude, les citoyens semblent sceptiques sur l'influence de leur participation et de leurs commentaires lors des séances d'information ou de consultation. Du côté des politiciens, on a tendance à ramener les commentaires négatifs émis en consultation comme étant non représentatifs de la population dans son ensemble ou comme constituant 
le prolongement de l'univers des débats partisans, univers au sein duquel les dissensions sont normales, voire intentionnelles. Dans le cas du dossier du Phare, le maire Labeaume affirme qu'il est au diapason de la population, laquelle «majorité silencieuse » sera d'avis contraire aux propos critiques exprimés sur ce projet urbain à l'occasion des séances de consultation publique.

Troisièmement, en concordance avec les constats précédents, les études techniques de faisabilité ou d'impact sont maintes fois absentes ou peu considérées dans le processus décisionnel. Dans une société qui parait fatiguée des débats, où chacun se replie sur son quant-à-soi, les projets soulèvent une pléthore d'avis et de commentaires qui se valent tous plus ou moins, aux yeux de nombre de citoyens. Il s'agit d'une énième controverse pour le citoyen-contribuable surinformé, qui a fréquemment pour critère suprême d'analyse l'effet pressenti sur son compte d'impôts fonciers.

Dans un sens, l'urbanisme cesse d'être de la planification pour redevenir un art urbain. Selon cette perspective, les goûts et les couleurs ne se discutent pas, et les opinions se côtoient sans grande possibilité d'argumentation ou d'arbitrage. Les pionniers de l'urbanisme ont pourtant souligné très tôt que toute intervention doit être fondée sur une connaissance approfondie du milieu d'accueil et ne plus être vue comme un acte individuel de création.

En dernier lieu, les projets urbains de Québec ont ramené sur le tapis le questionnement sur la scientificité de l'urbanisme. Les experts en la matière peuvent-ils réellement prévoir les conséquences d'une intervention? Bien sûr, il n'y a pas de laboratoire pour reproduire une dynamique urbaine à petite échelle, pour observer les réactions du système et pour en tirer des conclusions. Toutefois, ce caractère scientifique incomplet de la discipline ne devrait pas servir de justification à l'absence d'analyses prévisionnelles. Une étude imparfaite vaut probablement mieux qu'une absence complète de réflexions documentées. L'histoire de l'urbanisme, constituée d'essais et d'erreurs, est en soi une source de connaissances de grande valeur, pour autant qu'on s'y abreuve.

Au final, la réalité sur le terrain met à l'épreuve les principes de base de l'urbanisme et, dans le cas présent, devient une source d'enseignement sur le nouveau contexte d'exercice de la profession. Cependant, les dysfonctionnements observés seraient intrinsèques au fonctionnement de la gouvernance locale, de manière générale, et pas spécifiquement aux pratiques des professionnels attitrés. Sans l'ombre d'un doute, on n'est plus à l'époque de l'urbanisme professionnel triomphant, la discipline ayant depuis longtemps fait acte de contrition. L'humilité actuelle des urbanistes est le résultat des insuccès du passé autant que de la multitude de critiques provenant notamment des universitaires des sciences sociales, au cours des dernières décennies. Néanmoins, la montée des politiciens populistes ou entrepreneurs aux opinions bien arrêtées va visiblement à l'encontre de cette leçon de l'histoire en faveur de réflexions mûries et nuancées. De toute évidence, l'urbanisme restera toujours assujetti au politique, pour le meilleur et pour le pire. Les dilemmes de Québec ne font que rappeler cette réalité aux acteurs et observateurs de la scène locale.

\section{NOTES}

1 La fontaine de Tourny, l'Espace 400e, les travaux au pourtour du bassin Louise, la promenade Samuel-De Champlain, etc.

2 D'autres enjeux sont aussi en mouvement, notamment l'avenir des terres agricoles des Sœurs de la Charité à Beauport ou le réaménagement du quartier militaire à Sainte-Foy, mais ceux-ci nous semblent moins importants en matière de conséquences à l'échelle de l'agglomération.

3 À moins d'avis contraire, le terme agglomération est utilisé ici pour désigner une zone urbaine de grande taille intégrant une ville centre et les banlieues qui l'entourent. Cela diffère du terme administratif d'agglomération, qui est une instance supralocale créée par le gouvernement du Québec lors de la réorganisation des municipalités du Québec de 2000-2006.

4 La RMR de Québec comprend les Villes de Québec et de Lévis ainsi que 26 autres municipalités adjacentes de plus petites tailles. La RMR n'est pas une instance administrative. Cependant, la Communauté métropolitaine de Québec (CMQ) est un organisme supramunicipal qui a adopté le découpage territorial de la RMR pour exercer ses fonctions de planification régionale.

5 Dans le contexte canadien, notons cependant que la RMR d'Halifax, qui est peuplée de 403390 personnes, présente un pourcentage comparable à celui de Québec, soit $88,7 \%$ de non-immigrants. 
6 Deux municipalités se sont soustraites au regroupement ou reconstituées en 2006, soit L'Ancienne-Lorette et Saint-Augustin-deDesmaures.

7 Le BAPE est un organisme semi-indépendant mis en place par le gouvernement du Québec en 1978. Il organise et gère des consultations et des évaluations d'impact principalement sur les projets d'infrastructures ou industriels si ceux-ci répondent à certains critères de taille et/ou à la demande de citoyens. Il fait ensuite des recommandations au ministre de l'Environnement et de la Lutte contre les changements climatiques.

8 Documentation repérée à www.reseaustructurant.info/evenements/\#informations-juin

9 Ces pôles d'emplois sont principalement le siège social des Caisses populaires Desjardins, la raffinerie Jean-Gaulin d’Énergie Valero ainsi que les chantiers maritimes de la Davie.

10 Les données plus récentes de l'enquête Origine-Destination 2017 à Québec et Lévis ont été rendues disponibles en mai 2019. Repéré à www.ville.quebec.qc.ca/apropos/planification-orientations/transport/enquete-origine-destination.aspx

11 Selon la même source, la part du transport en commun est de $11,1 \%$, alors que $6,3 \%$ des gens se déplaçaient à pied et $1,3 \%$ à bicyclette.

12 Repéré à http://legisquebec.gouv.qc.ca/fr/ShowDoc/cs/C-11.5

13 Le promoteur a aussi tenu des séances d'information auprès des citoyens au printemps 2018.

14 Pour plus de détails à ce sujet, voir le site Internet suivant : www.ville.quebec.qc.ca/apropos/planification-orientations/ transport/consultations.aspx

15 Ironiquement, la loi provinciale qui oblige les référendums lors d'une modification de zonage a été récemment modifiée et, à cette occasion, le maire Labeaume avait qualifié l’abandon des référendums de pratique antidémocratique.

16 Repéré à www.ville.quebec.qc.ca/citoyens/participation-citoyenne/politique-de-participation-publique/index.aspx

\section{RÉFÉRENCES}

Baché, M.-H. et Gauthier, M. (2011). Participation, urbanisme et études urbaines : quatre décennies de débats et d'expériences depuis $A$ ladder of citizen participation de S.R. Arnstein. Participations, 1(1), 36-66. doi: 10.3917/parti.001.0036.

Beauregard, R. (2013). The neglected places of practice. Planning Theory and Practice, 14(1), 8-19. doi: 10.1080/14649357.2012.744460

Béland, G. (2017, 16 décembre). «Québec est la ville parfaite pour le tramway », dit Richard Bergeron. La Presse. Repéré à www.lapresse.ca/actualites/regional/201712/15/01-5147360-quebec-est-la-ville-parfaite-pour-le-tramway-ditrichard-bergeron.php

Belley, S., Quesnel, L. et Villeneuve, P. (2011). Québec 2009 : l'impact de la fierté retrouvée sur le pouvoir local. Dans S. Breux, L. Béhrer et J.-P. Collin (Éd.) Portraits de la démocratie urbaine an Québec : les élections municipales de 2009 (pp. 145-171). Québec, QC : Presses de l'Université Laval.

Boisvert, L. (2018a, 17 mars). Québec dévoile son projet de tramway. Société Radio-Canada. Repéré à https://ici.radiocanada.ca/nouvelle/1089685/tramway-quebec-transport-en-commun-projet-transport-structurant

Boisvert, L. (2018b, 24 novembre). Consultation à Sainte-Foy : du meilleur au pire. Société Radio-Canada. Repéré à https://ici.radio-canada.ca/nouvelle/1137736/phare-projet-exception-exige-consultation-exception

Borde, V. (2017, 7 décembre). La folle idée du troisième lien Québec-Lévis. L'Actualité. Repéré à https:/ /lactualite.com/santeet-science/2017/12/07/la-folle-idee-du-troisieme-lien-quebec-levis

Bourque, F. (2019, 31 janvier). Pourquoi les immigrants boudent-ils Québec? Le Soleil. Repéré à www.lequotidien.com/ chroniques/francois-bourque/pourquoi-les-immigrants-boudent-ils-quebec-92a8bee8a73cc7d3a39ddeb541e9212c

Bourque, F. (2018a, 3 novembre). Québec capitule. Le Soleil. Repéré à www.lesoleil.com/chroniques/quebec-capitule19efc00963b145424b9d8b117cf42316

Bourque, F. (2018b, 22 octobre). Une astuce légale pour forcer le Phare. Le Soleil. Repéré à www.lesoleil.com/chroniques/ francois-bourque/ une-astuce-legale-pour-forcer-le-phare-5916f536445254f22a048bc692a355c0

Bourque, F. (2018c, 16 octobre). Le bulldozer du Phare en marche. Le Soleil. Repéré à www.lesoleil.com/chroniques/francoisbourque/le-bulldozer-du-phare-en-marche-cf0851d6107c5d3830ba789cffab7262 
Bourque, F. (2015, 31 janvier). Les mirages de la tour-phare. Le Soleil. Repéré à www.lesoleil.com/archives/les-mirages-de-latour-phare-bdf129f22d8887a73b172d30fe0841fb

Boutin, M. (2008). 30 ans d'urbanisme à Québec : une ville marquée par un traumatisme qui n'est pas celui qu'on pense. Revue Inter, 100, 34-39. Repéré à www.erudit.org/fr/revues/inter/2008-n100-inter1112152/45509ac.pdf

Boyer (2019). Québec : enclave francophone authentique (et surréaliste) en Amérique du Nord. Le Soleil. Repéré à www.lesoleil.com/opinions/quebec-enclave-francophone-authentique-et-surrealiste-en-amerique-du-nord0c9862ef95dc4b3448a25fbc8ea0e234?utm_\%20source=dlvr.it\&utm_medium=twitter

Cardinal, F. (2015, 25 février). Une tour qu’on regrettera. La Presse. Repéré à www.lapresse.ca/debats/chroniques/francoiscardinal/201502/23/01-4846518-le-phare-une-tour-quon-regrettera.php

Debrie, J. (2018). D'un problème d'action publique à la structuration d'un champ de recherche... et vice-versa : l'exemple de l'introduction de la question logistique dans l'aménagement urbain. Métropoles, 23. Repéré à http://journals. openedition.org/metropoles/6601

Demazière, C. (2015). Les enjeux de la planification spatiale en Angleterre et en France : regards croisés. Espaces et sociétés, 160-161(1), 67-84. Repéré à www.cairn.info/revue-espaces-et-societes-2015-1-page-67.htm

Demers, C., Després, C., Dufaux, F., Potvin, A., Vachon, G., Fortin, A., Morin, D., Boulianne, M., Gauthier, P. et Lord, S. (2015, 27 février). Le Phare, un colosse aux pieds d'argile. Le Devoir. Repéré à www.ledevoir.com/ opinion/idees/432974/projet-immobilier-geant-a-quebec-le-phare-un-colosse-au-pied-d-argile

Dorning, M. A., Koch, J., Shoemaker, D. A. et Meentemeyer, R. K. (2015). Simulating urbanization scenarios reveals tradeoffs between conservation planning strategies. Landscape and Urban Planning, 136, 28-39. doi: 10.1016/j.landurbplan.2014.11.011

Douay, N. (2013). La planification urbaine française : théories, normes juridiques et défis pour la pratique. L'Information géographique, 77(3), 45-70. Repéré à www.cairn.info/revue-l-information-geographique-2013-3-page-45.htm

Dupuis, J. (2013). Le pont Pierre-Laporte, un exploit du génie québécois. Portail Constructo. Repéré à www.portailconstructo.com/ infoconstructo/ pont_pierre_laporte_exploit_genie_quebecois

Fainstein, S. S. et Defilippis, J. (2016). The structure and debates of planning theory. Dans S. S. Fainstein et J. DeFilippis (dir.). Readings in planning theory (4e éd., pp. 1-18). Cambridge, MA: Blackwell.

Faugier, É. (2009). Automobile, transports urbains et mutations : l'automobilisation urbaine de Québec, 1919-1939. Revue d'bistoire urbaine, 38(1), 26-37. doi: 10.7202/038465ar

Fischler, R. (juillet-décembre 2015). Cinquante thèses sur l'urbanisme et les urbanistes. Revue internationale d'urbanisme, 1. Repéré à http:/ / riurba.net/Revue/ cinquante-theses-sur-lurbanisme-et-les-urbanistes

Fortin, P.-O. (2016, 25 octobre). Le trafic Québec-Lévis en 8 chiffres. Journal de Québec. Repéré à www.journalde quebec.com/2016/10/25/le-trafic-quebec-levis-en-8-chiffres

Gagné, L. (2018a, 15 novembre). Catherine Dorion compare le 3e lien à « une ligne de coke ». Société Radio-Canada. Repéré à https://ici.radio-canada.ca/nouvelle/1136060/deputee-quebec-solidaire-catherine-dorion-compare-troisiemelien-ligne-de-coke

Gagné, L. (2018b, 22 novembre). Projet Le Phare : la privatisation du plan d’urbanisme dénoncée. Société Radio-Canada. Repéré à https://ici.radio-canada.ca/nouvelle/1137386/seance-consultation-publique-projet-le-phare-plan-urbanismequebec-groupe-dallaire

Gagnon, J.-M. (2018, 14 août). 5 corridors potentiels pour un 3e lien Québec-Lévis. Le Journal de Québec. Repéré à www.journaldequebec.com/2018/08/14/cinq-corridors-potentiels-seront-etudies-pour-un-troisieme-lien-quebeclevis

Geronimi, M. (2008). Québec et la Nouvelle-Orléans : paysages imaginaires français en Amérique du Nord. Paris : Bélin.

Grandmont, É. (2018, 16 novembre). Étalement urbain à Lévis : une mise au point s’impose. Le Soleil.

Repéré à www.lesoleil.com/opinions/point-de-vue/etalement-urbain-a-levis--une-mise-au-point-simpose-fecddc6b67b8234a42ea37133b46ebdf

Grenier, F. (2008). Raoul Blanchard : Québec sous la loupe d'un grand géographe. Cap-aux-Diamants, 92, 25-29.

Repéré à www.erudit.org/fr/revues/cd/2008-n92-cd1045684/7143ac/ 
Groupe Dallaire. (2018). Le Phare. Repéré à https://lephareqc.ca

Guy, S. et Henneberry, J. (2000). Understanding urban development processes: Integrating the economic and the social in property research. Urban Studies, 37(13), 2399-2416. doi: 10.1080/00420980020005398

Hodge, G. et Gordon, D. (2013). Planning Canadian communities (6e éd.). Toronto, ON : Nelson.

Hulbert, F. (1994). Essai de géopolitique urbaine et régionale : la comédie urbaine de Québec (2e éd.). Québec, QC : Méridien.

La Presse canadienne (2019, 25 février). Une étude minimise la congestion routière à Québec. Le Soleil. Repéré à www.lesoleil.com/actualite/une-etude-minimise-la-congestion-routiere-a-quebec-2c1ec18d4f5c1d57a3f8c2586e185c35

Lavallée, J.-L. (2017, 13 avril). Québec, la seule grande ville canadienne sans projet de transport collectif. Le Journal de Québec. Repéré à www.tvanouvelles.ca/2017/11/13/quebec-la-seule-ville-canadienne-sans-projet-de-transport-collectif

Lebel, J. (2005). « Le Québec se modernise! » : les années Lamontagne. Cap-aux-Diamants, hors-série, 65-69. Repéré à www.erudit.org/fr/revues/cd/2005-cd1045177/518ac.pdf

Leclerc, Y. (2018). L'action culturelle et le développement territorial : le quartier Saint-Roch à Québec et autres cas de réussite. Québec, QC : Presses de l’Université Laval.

L’Hébreux, M. (2008). Le pont de Québec (2e éd.). Québec, QC : Septentrion.

Lois González, R. C., Pazos Otón, M. et Wolff, J.-P. (2013). Le tramway entre politique de transport et outil de réhabilitation urbanistique dans quelques pays européens : Allemagne, Espagne, France et Suisse. Annales de géographie, 694(6), 619-643. Repéré à www.cairn.info/revue-annales-de-geographie-2013-6-page-619.htmEn

Mercier, G. (2010). La modernisation de Québec après la Seconde Guerre mondiale : une ville sous l'emprise de sa propre image. Dans L. K. Morrisset (dir.). La ville, phénomène de représentation (pp. 121-144). Québec, QC : Presses de l’Université du Québec.

Mercier, G. (2000). L'urbanisme des échecs urbanistiques : la rhétorique du renouveau du quartier Saint-Roch à Québec depuis 1990. Dans G. Sénécal et D. Saint-Laurent (dir.). Les espaces dégradés (pp. 101-115). Québec, QC : Presses de l'Université du Québec.

Mercier, G. et Roy, F. (2014). La centralité urbaine à Québec : un grand projet en continuel renouvellement. Organisations et territoires, 23(3), 55-64. doi: 10.1522/revueot.v23i3.142.

Ministère des Transports du Québec (MTQ), Réseau de transport de la Capitale (RTC), Société de transport de Lévis (STL) et Communauté métropolitaine de Québec (CMQ). (2015). Enquête Origine-Destination 2011 sur la mobilité des personnes dans la région de Québec - Volet Enquête-ménages : sommaire des résultats. Repéré à www.transports.gouv.qc.ca/fr/ministere/accesinformation-renseignements-personnels/documents-reglement-diffusion/Documents/etudes/recherches-relatives-3elien/Enquete-OD-2011.pdf

Moalla, T. (2019, 31 janvier). Tramway : Labeaume souhaite un BAPE «très bien circonscrit ». Le Journal de Québec. Repéré à www.journaldemontreal.com/2019/01/31/tramway-labeaume-veut-un-bape-tres-bien-circonscrit

Moalla, T. (2018a, 2 octobre). Legault réaffirme son engagement pour le troisième lien et le tramway. Le Journal de Québec. Repéré à www.journaldequebec.com/2018/10/02/legault-reitere-son-engagement-pour-le-troisieme-lien-et-le-tramway

Moalla, T. (2018b, 29 octobre). Les 65 étages du Phare au cœur des échanges très contrastés. Le Journal de Québec. Repéré à www.journaldequebec.com/2018/10/29/opinions-tres-contrastees-sur-le-phare

Morin, A. (2016, 28 octobre). Labeaume pour un troisième lien à l'ouest. Le Soleil. Repéré à www.lesoleil.com/ actualite/labeaume-pour-un-troisieme-lien-a-louest-fe8d4a2f102d4bb29434acc4bae86189

Morin, A. (2013, 9 septembre). Congestion : la solution de Labeaume passe par l'auto... avant le tramway. Le Soleil. Repéré à www.lapresse.ca/le-soleil/actualites/transports/201309/09/01-4687615-congestion-la-solution-de-labeaumepasse-par-lauto-avant-le-tramway.php

Morin, A. et Gaudreau, V. (2015, 2 mars). Québec et Lévis visent un service rapide par bus de 38 km. Le Soleil. Repéré à www.lesoleil.com/actualite/quebec-et-levis-visent-un-service-rapide-par-bus-de-38-km36bcf7c64c216fb398304676cd4ebff4

Néron, J.-F. (2019, 2 mars). Enquête origine-destination : conclusions « hasardeuses ». Le Soleil. Repéré à www.lesoleil.com/ actualite/enquete-origine-destination-conclusions-hasardeuses-video-421ad1d028805f47920d9e8fb37a53de 
Noppen, L., Morisset, L. K. et Karam, H. (2008). Québec : le génie du lieu. Cap-aux-Diamants, 93, 25-43. Repéré à www.erudit.org/fr/revues/cd/2008-n93-cd1045841/6889ac/

Pelchat, P. (2009, 16 mars). Trafic sur les autoroutes de la région de Québec : hausses de plus de 30 \%. Le Soleil. Repéré à www.lesoleil.com/actualite/la-capitale/trafic-sur-les-autoroutes-de-la-region-de-quebec-hausses-de-plus-de-30-7c310f15ad084f664c3189b0e6ffd11a

Polèse, M. (2012). L'autre « mystère de Québec » : regards sur une mutation économique étonnante. Recherches sociographiques, 53(1), 133-156. doi: 10.7202/1008922ar

Porter, I. (2019, 22 janvier). Québec veut éviter une étude du BAPE sur son projet de tramway. Le Devoir. Repéré à www.ledevoir.com/politique/ville-de-quebec/546005/la-ville-de-quebec-ne-souhaite-pas-de-bape-sur-le-tramway

Porter, I. (2018a, 17 mars). Un tramway nommé désir de modernité et de mobilité. Le Devoir. Repéré à www.ledevoir.com/ politique/ville-de-quebec/522916/tramway-quebec

Porter, I. (2018b, 30 octobre). Projet Le Phare à Québec : les 65 étages dérangent. Le Devoir. Repéré à www.ledevoir.com/ societe/540193/projet-le-phare-les-65-etages-derangent

Porter, I. (2018c, 22 décembre). Régis Labeaume tourne la page d'une année difficile. Le Devoir. Repéré à www.ledevoir.com/politique/ville-de-quebec/544182/labeaume-tourne-la-page-d-une-annee-difficile

Racine, J.-B. et Villeneuve, P. (1992). Canada, Collection Géographie universelle. Paris : Hachette/Reclus.

Réseau de transport de la Capitale (2003). Le tramway de Québec : une nouvelle vision de la mobilité urbaine - Étude d'opportunité et de faisabilité d'un système léger sur rail dans l'agglomération de la capitale. Québec, QC : RTC. Repéré à www.rtcquebec.ca/ Portals/0/Pages/A_Propos_Du_RTC/Publications/Doc/RapportTramway_Quebec_2003.pdf

Robitaille, A. (2015, 21 février). Carences phares. Le Devoir. Repéré à www.ledevoir.com/opinion/editoriaux/432518/tour-de65-etages-a-quebec-carences-phares

Roseland, M. (2012). Toward sustainable communities: Solutions for citizens and their governments (4e éd.). Vancouver, BC : New Society Publishers.

Roudil, R. et Roudil, N. (2012). Fabriquer la ville à l’heure de l'injonction au durable. Métropolitiques. Repéré à www.metropolitiques.eu/Fabriquer-la-ville-a-l-heure-de-l.html

St-Onge, S. (2018, 2 novembre). Labeaume réitère son appui au Phare. Le Soleil. Repéré à www.lesoleil.com/actualite/ la-capitale/labeaume-reitere-son-appui-au-phare-6fc8f981e8ee06b17afcabf8f34b18a7

Simard, P. (2018, 3 avril). Le tramway et le troisième lien ne peuvent cohabiter. Le Devoir. Repéré à www.ledevoir.com/ opinion/libre-opinion/524264/le-tramway-et-le-troisieme-lien-ne-peuvent-cohabiter

Simard, M. (2003). La participation à l'aménagement ou le difficile exercice de la démocratie locale. Dans J. Désy et collab. (dir.). Le GRIR : 20 ans de recherche et d'intervention (pp. 415-441). Saguenay, QC : Groupe de recherche et d'intervention régionales.

Siou, M.-M. (2017, 20 novembre). Le Vieux-Québec, «made in China ». Le Devoir. Repéré à www.ledevoir.com/societe/ transports-urbanisme/513408/le-vieux-quebec-made-in-china

Société canadienne d'hypothèques et de logement (SCHL). (2018). Perspectives du marché de l'habitation : région du Québec. Ottawa, $\mathrm{ON}$ : SCHL. Repéré à www.cmhc-schl.gc.ca/fr/data-and-research/publications-and-reports/housing-market-outlookhighlights

Société Radio-Canada (SRC). (2018). Québec dévoile son projet de tramway. Repéré à ici.radio-canada.ca/nouvelle/1089685/ tramway-quebec-transport-en-commun-projet-transport-structurant

Statistique Canada. (2016a). Recensement de la population, Ottawa, ON : Statistique Canada. www12.statcan.gc.ca/censusrecensement/geo/maps-cartes/pdf/ S0503/2016S0503421.pdf

Statistique Canada. (2016b). Série «Perspective géographique 》 - Recensement de 2016 : Québec (RMR) - Québec. Ottawa, ON : Statistique Canada. Repéré à www12.statcan.gc.ca/census-recensement/2016/as-sa/fogs-spg/Facts-CMAfra.cfm?TOPIC $=5 \& \mathrm{~L} A N G=$ fra\&GK $=\mathrm{CMA} \& \mathrm{GC}=421$

Statistique Canada. (2016c). Caractéristiques de la population active selon la région métropolitaine de recensement, moyennes mobiles de trois mois, données désaisonnalisées et non désaisonnalisées, 5 derniers mois. 
Ottawa, ON : Statistique Canada. Repéré à www150.statcan.gc.ca/t1/tbl1/fr/tv.action?pid=1410029401

Vallières, M. (2010). Québec : histoire en bref. Québec, QC : Presses de l’Université Laval.

Verville, A. (2018, 12 novembre). En urbanisme, les conditions de laboratoire n'existent pas. Le Soleil.

Repéré à www.lesoleil.com/opinions/point-de-vue/en-urbanisme-les-conditions-de-laboratoire-nexistent-pas927a5ea230bf0a712299b726031c9a36

Ville de Québec. (2019). Secteur est | Avancement du projet - 11 juin 2019 : présentation. Repéré à http://www.reseaustructurant.info/docs/presentations/RST-TR-EST-PR-PUBLIC-2019-06-11.pdf

Ville de Québec (2012). Programme particulier d'urbanisme du plateau centre de Sainte-Foy. Québec, QC : Ville de Québec, Service de l’aménagement du territoire. Repéré à www.ville.quebec.qc.ca/apropos/planification-orientations/amenagement_ urbain/ppu/ppu_saintefoy/docs/ppu_plateau_centre_sainte_foy_edit\%C3\%A9.pdf

Ville de Québec (2011). Plan de mobilité durale : pour viorre et se déplacer autrement. Rapport du Groupe de travail sur la mobilité durable. Québec, QC : Ville de Québec. Repéré à www.ville.quebec.qc.ca/apropos/planification-orientations/ transport/docs/PlanMobiliteDurable.pdf 